\title{
Antimicrobial Resistance Patterns in Clinically Significant Isolates from Medical Wards of a Tertiary Care Hospital in North India
}

\author{
Venkatesh Sudharsan Vaithiyam ${ }^{1, \odot}$ Neha Rastogi ${ }^{2} \quad$ Piyush Ranjan ${ }^{1, \odot} \quad$ Niranjan Mahishi ${ }^{2} \quad$ Arti Kapil $^{3}$ \\ Sada Nand Dwivedi ${ }^{4}$ Manish Soneja ${ }^{1} \quad$ Naveet Wig ${ }^{1} \quad$ Ashutosh Biswas $^{1}$
}

${ }^{1}$ Department of Medicine, All India Institute of Medical Sciences, New Delhi, India

2Department of Medicine and Microbiology, All India Institute of Medical Sciences, New Delhi, India

${ }^{3}$ Department of Microbiology, All India Institute of Medical

Sciences, New Delhi, India

${ }^{4}$ Department of Biostatistics, All India Institute of Medical Sciences,

New Delhi, India

J Lab Physicians:2020;12:196-202 Address for correspondence Piyush Ranjan, MD, Department of
Medicine, All India Institute of Medical Sciences, New Delhi, 110029,
India (e-mail: drpiyushaiims@gmail.com; drpiyushdost@gmail.com).

\begin{abstract}
Keywords

- drug-resistant infections

- antimicrobial resistance, antibiogram
\end{abstract}

Background The global burden of infections due to multidrug-resistant organism (MDRO) has a significant impact on patients' morbidity and mortality along with increased healthcare expenditure.

Aim This article estimates the prevalence of MDRO and the spectrum of clinical infectious syndromes caused by these organisms in medical wards of a tertiary care hospital in India.

Design and Methods A cross-sectional observational study was performed among patients admitted in medicine wards diagnosed with the various infectious syndromes and one or more clinically significant positive culture at a tertiary care hospital in North India over a period of 18 months.

Results Out of 323 clinically significant microbiological culture isolates from 229 patients included in the study, $86(27 \%)$ isolates showed multidrug resistance (MDR) pattern, 197 (61\%) isolates showed possible extremely drug-resistance pattern, and only $40(12 \%)$ isolates showed nonmultidrug-resistance pattern of antibiogram.

Conclusion The prevalence of MRDOs is high in clinically significant culture isolates from medicine wards in India. This emphasizes the importance of appropriate antibiotic usage and implementation of antibiotic stewardship programs in this part of the world.

\section{Introduction}

Antimicrobial resistance has assumed a "global threat," and according to a recently published report by World Health Organization, it reported that approximately 700,000 people die each year due to drug-resistant infections. ${ }^{1}$ Multidrug-resistant organisms (MDROs) lead to a wide spectrum of clinical

DOI https://doi.org/ $10.1055 / \mathrm{s}-0040-1721161$ ISSN 0974-2727. infections which poses a significant burden on healthcare systems by increasing patient's hospital stay, health care expenses, morbidity, and mortality. ${ }^{2}$

The emergence of antimicrobial resistance is a dynamic phenomenon and its successful containment mandates a periodic and updated assessment of antimicrobial susceptibility patterns in pathogens. ${ }^{3}$ The resistance pattern of different
(C) 2020. The Indian Association of Laboratory Physicians.

This is an open access article published by Thieme under the terms of the Creative Commons Attribution-NonDerivative-NonCommercial-License, permitting copying and reproduction so long as the original work is given appropriate credit. Contents may not be used for commercial purposes, or adapted, remixed, transformed or built upon. (https://creativecommons.org/licenses/by-nc-nd/4.0/)

Thieme Medical and Scientific Publishers Pvt. Ltd., A-12, 2nd Floor, Sector 2, Noida-201301 UP, India 
microorganisms varies in different parts of the world which is largely affected by the rational antimicrobial usage in that region. The utilization of antimicrobials is generally unregulated in developing countries like India. ${ }^{4}$ This is partly due to easy availability of antibiotics as over-the-counter drugs, lack of awareness on antibiotic resistance among health care workers, unregulated use of antibiotics in other fields like in animal sciences, and the paucity of studies related to antimicrobial surveillance and antibiotic resistance patterns in a different spectrum of infections. Regular monitoring of local trends of antibiotic resistance detects early shifts in susceptibility and also serves as a basis for empirical therapy, formulary decisions, and infection control practices. ${ }^{5}$ Periodic update of antimicrobial resistance patterns from the different parts of the world can be instrumental in preventing the evolution of "superbugs." Therefore, studies on antibiotic resistance may prove to be instrumental in forming guidelines for appropriate empiric antimicrobial therapy especially in countries bearing a heavy burden of drug resistance. These guidelines may be initially used to form as intrahospital policy on antibiotic usage, and further in accordance with other regional and national health data can be utilized for formulation of national antibiotic policy. Considering this emerging national and global crisis, we undertook the present study to estimate the burden of infections caused by MDROs in an Indian setup. We also formulated an antibiogram based on the local sensitivity patterns to guide antimicrobial prescription practices.

\section{Methodology}

This cross-sectional observational study was conducted in the department of medicine in an apex teaching medical institute of Northern India. Despite being a tertiary care center, patients can visit the institute for consultation in outpatient department (OPD) and/or emergency department, even without any prior referral. Patients are admitted in medicine wards and intensive care units (ICUs) via the medicine OPD and emergency department and represent a highly heterogeneous group presenting with a varied spectrum and severity of infections. The study was approved by the institute's ethics review board and informed consent was obtained from the participants before enrolment.

The patients admitted to the medical wards and ICUs of the department of medicine over a period of 18 months (August 2017-February 2019) were screened for inclusion in the study. All admitted patients were examined for community-acquired or hospital-acquired infectious syndromes. All clinically relevant samples like endotracheal aspirate, mini-bronchoalveolar lavage (BAL), sputum, blood, urine, cerebrospinal fluid, and other body fluids were obtained from patients with clinical suspicion of any infectious syndromes. Samples were sent to the microbiology laboratory by the treating physician as per requirement in sterile containers, except for blood which was inoculated manually in blood culture bottles at the patient's bedside. Sample processing, direct demonstration of organisms, inoculation, and identification were done as per standard microbiological practices. Patients with infectious syndrome with at least one clinically significant culture growth were enrolled in the study. For assessment of antibiotic resistance patterns and development of an antibiogram, all samples with one or more clinically significant culture-positive isolate were included in the study. Samples with contaminant growth or commensal growth on culture were excluded from the study. Single sample is usually considered in each patient, but multiple samples from same patient were only considered in two scenarios: (1) When the samples were obtained from same site (i.e., BAL), but from different clinical infectious episode. This is not unusual in patients who have long hospital course. (2) If samples were obtained from same patients from two different sites (i.e., two different clinical infectious syndromes).

\section{Antimicrobial Susceptibility Testing}

Antimicrobial susceptibility was done using the disc diffusion method (Kirby-Bauer test) and interpretation was done in accordance with recent Clinical and Laboratory Standards Institute (CLSI; CLSI -M100 guidelines ${ }^{6}$ ) Antimicrobial agents analyzed in our study included amikacin, gentamicin, amoxicillin/clavulanic acid, ciprofloxacin, ceftriaxone, imipenem,meropenem, and piperacillin/tazobactam for Gram-negative bacterial isolates. For Gram-positive bacterial isolates penicillin, amoxicillin-clavulanic acid, cefoxitin (surrogate marker of methicillin resistance), amikacin, gentamicin, linezolid, teicoplanin, and co-trimoxazole were tested. Vancomycin susceptibility in Gram-positive and colistin susceptibility in Gram-negative isolates was assessed by microbroth dilution in accordance with the CLSI guidelines.

Various infectious syndromes like ventilator-associated pneumonia (VAP), hospital-acquired pneumonia (HAP), symptomatic and catheter-associated urinary tract infection (S-UTI, CA-UTI), primary and secondary bloodstream infections (BSIs), and others were defined as per the Centers for Disease Control and Prevention (CDC) National Healthcare Safety Network surveillance guidelines. ${ }^{7}$

\section{Definition of Drug Resistance}

As per the resistance patterns obtained and assessed using clinical breakpoints provided by the CLSI, the isolates were stratified into various resistance patterns of multidrug resistance (MDR). ${ }^{6} \mathrm{~A}$ bacterial isolate was considered nonsusceptible to an antimicrobial agent when it tested resistant or intermediate susceptible when using clinical breakpoints as interpretive criteria, as provided by the CLSI guidelines and European CDC. MDR was defined as nonsusceptibility to at least one agent in three or more antimicrobial categories. ${ }^{8}$ Extensive drug resistance (XDR) was defined as nonsusceptibility to at least one agent in all but two or fewer antimicrobial categories (i.e., bacterial isolates remain susceptible to only one or two categories). Pan drug resistance (PDR) was defined as nonsusceptibility to all agents in all antimicrobial categories (i.e., no agents tested as susceptible to that organism). ${ }^{8}$ However, in the majority of microbiological laboratories in resource-limited settings, resistance testing is done using limited and commonly used antibiotics. Hence, the terminology of "possible XDR" (P-XDR) was 
coined. Bacterial isolates were defined as "P-XDR" when found resistant to most of the routinely tested classes (more than three) of antimicrobials and susceptible to only one or two available and tested categories of antimicrobials. P-XDR, however, should still be regarded as a marker of extensive resistance. All isolates which were not satisfying the criteria for MDR and PDR were classified as nonmultidrug-resistant (NMDR) isolates. ${ }^{8}$

\section{Statistical Analysis}

Appropriate statistical analysis was done using univariate analysis (SPSS 17) to ascertain the significance of the acquisition of MDR/P-XDR isolates. A p-value of less than 0.05 was considered statistically significant.

\section{Results}

A total of 2,998 clinical samples from various patients with different infectious syndromes were obtained and subjected to microbiological culture for establishing a definitive etiological diagnosis. Of the 2,998 samples tested for culture and sensitivity, 686 yielded significant monomicrobial growth. Of these, 323 cultures from 229 patients were included for further analysis (363 cultures were excluded as they were either from the same clinical event or clinically insignificant).

\section{Spectrum of Infections}

Spectrum of infectious syndromes in the recruited patients and microbial pathogens isolated is given in - Table $\mathbf{1}$. Respiratory tract infections were the most common infection (43\%) followed by UTIs (24\%). Among respiratory tract infections, VAP (68.3\%) was the most common entity followed by $\operatorname{HAP}(23 \%)$ and CAP (3\%).

Microbiological analysis of 323 culture-positive bacterial isolates revealed the presence of both Gram-positive and Gram-negative pathogens with significant predominance of Gram-negative pathogens (92\%) (-Table 1). Acinetobacter baumannii (68\%) and Klebsiella pneumoniae (35\%) were the most common Gram-negative pathogens mainly isolated from respiratory samples and enterococcus species and Staphylococcus aureus were commonly isolated Gram-positive pathogens (-Table 1). Among UTIs, Escherichia coli (52.4\%) was the most common organism isolated both in S-UTI and CA-UTI.

\section{Antimicrobial Resistance Patterns}

We encountered perturbing prevalence of antimicrobial resistance among culture isolates. Approximately $90 \%$ of the culture isolates had significant antimicrobial resistance with 27 (86) and 61\% (197) isolates showing MDR and P-XDR type of antimicrobial resistance, respectively, in - Table 2. A. baumannii and K. pneumoniae, the most

Table 1 Distribution of pathogens in different spectrum of infections $(n=323)$

\begin{tabular}{|c|c|c|c|c|c|c|c|c|c|}
\hline \multirow{2}{*}{$\begin{array}{l}\text { Sr. } \\
\text { no. }\end{array}$} & \multirow[t]{2}{*}{ Infection } & \multicolumn{8}{|c|}{ Isolates } \\
\hline & & $\begin{array}{l}\text { Acinetobacter } \\
\text { baumannii }\end{array}$ & $\begin{array}{l}\text { Klebsiella } \\
\text { pneumoniae }\end{array}$ & $\begin{array}{l}\text { Pseudomonas } \\
\text { aeruginosa }\end{array}$ & $\begin{array}{l}\text { Escherichia } \\
\text { coli }\end{array}$ & $\begin{array}{l}\text { Staphylococcus } \\
\text { aureus }\end{array}$ & $\begin{array}{l}\text { Enterococcus } \\
\text { faecium }\end{array}$ & Others & Total (\%) \\
\hline \multirow[t]{5}{*}{ A } & 1. RTI & 68 & 36 & 15 & 13 & 0 & 1 & 6 & 139(43) \\
\hline & 1.1 CAP & 0 & 3 & 0 & 1 & 0 & 0 & 0 & $4(1.2)$ \\
\hline & 1.2 HAP & 12 & 6 & 6 & 7 & 0 & 0 & 1 & $32(9.9)$ \\
\hline & 1.3 VAP & 56 & 26 & 7 & 3 & 0 & 0 & 3 & $95(29.4)$ \\
\hline & $\begin{array}{l}1.4 \text { Pleural } \\
\text { effusion }\end{array}$ & 0 & 1 & 2 & 2 & 0 & 1 & 2 & $8(2.47)$ \\
\hline \multirow[t]{3}{*}{ B } & 2. UTI & 1 & 19 & 0 & 43 & 0 & 8 & 7 & $78(24.1)$ \\
\hline & $2.1 \mathrm{~S}-\mathrm{UTI}$ & 0 & 5 & 0 & 25 & 0 & 1 & 0 & $31(9.5)$ \\
\hline & 2.2 CA-UTI & 1 & 14 & 0 & 20 & 0 & 7 & 5 & 47 (14.5) \\
\hline \multirow[t]{4}{*}{ C. } & 3. BSI & 17 & 22 & 1 & 13 & 2 & 6 & 12 & $73(22.6)$ \\
\hline & 3.1 P-BSI & 5 & 8 & 0 & 10 & 0 & 5 & 2 & 30 (9.28) \\
\hline & $3.2 \mathrm{~S}-\mathrm{BSI}$ & 11 & 4 & 1 & 1 & 2 & 0 & 10 & 29 (8.97) \\
\hline & 3.3 CLABSI & 1 & 10 & 0 & 2 & 0 & 1 & 0 & $14(4.33)$ \\
\hline D. & 4. SSTI & 5 & 3 & 5 & 2 & 5 & 0 & 1 & 20 (6.19) \\
\hline \multirow[t]{5}{*}{ E. } & $\begin{array}{l}\text { 5. Sterile site } \\
\text { infection }\end{array}$ & 1 & 3 & 1 & 3 & 0 & 0 & 0 & $8(2.4)$ \\
\hline & 5.1 Meningitis & 1 & 0 & 1 & 2 & 0 & 0 & 0 & $4(1.2)$ \\
\hline & 5.2 Cholangitis & 0 & 1 & 0 & 0 & 0 & 0 & 0 & $1(0.3)$ \\
\hline & 5.3 Ascites & 0 & 2 & 0 & 1 & 0 & 0 & 0 & $3(0.9)$ \\
\hline & Total & 92 & 86 & 22 & 75 & 7 & 15 & 16 & 323 \\
\hline
\end{tabular}

Abbreviations: BSI, blood stream infections; CAP, community-acquired pneumonia; CA-UTI, catheter-associated urinary tract infection; CLABSI, central line-associated blood stream infection; HAP, hospital-acquired pneumonia; PBSI, primary blood stream infection; RTI, respiratory tract infections; S-BSI, secondary blood stream infection; SSTI, skin and soft tissue infection; UTI, urinary tract infection; S-UTI, symptomatic urinary tract infection; VAP, ventilator-associated pneumonia. 
Table 2 Drug resistance patterns among various culture isolates

\begin{tabular}{|c|c|c|c|c|c|}
\hline Bacteria & $\begin{array}{l}\text { Nonmultidrug } \\
\text { resistant } \\
\text { (NMDR), } n(\%)\end{array}$ & $\begin{array}{l}\text { Multidrug } \\
\text { resistant } \\
(\mathrm{MDR}), n(\%)\end{array}$ & $\begin{array}{l}\text { Possible extensive drug } \\
\text { resistant } \\
\text { (P-XDR), } n(\%)\end{array}$ & Total & $p$-Value \\
\hline A. baumannii & $1(1)$ & $9(10)$ & $82(89)$ & 92 & $<0.0001$ \\
\hline K. pneumoniae & $5(6)$ & $11(13)$ & $70(81)$ & 86 & $<0.0001$ \\
\hline P. aeruginosa & $6(27)$ & $2(9)$ & $14(64)$ & 22 & 0.032 \\
\hline E. coli & $12(16)$ & $42(56)$ & $21(28)$ & 75 & $<0.0001$ \\
\hline E. faecium & 0 & $10(66.67)$ & $5(33.33)$ & 15 & $<0.003$ \\
\hline E. faecalis & 1 & 0 & 1 & 2 & - \\
\hline S. aureus & $3(30)$ & $7(70)$ & 0 & 10 & 0.001 \\
\hline \multicolumn{6}{|l|}{ Others } \\
\hline Salmonella spp. & $9(90)$ & $1(10)$ & 0 & 10 & $<0.001$ \\
\hline Enterobacterspp. & 2 & 1 & 1 & 4 & 0.016 \\
\hline K. oxytoca & 1 & 1 & 1 & 3 & 0.295 \\
\hline B. серасіа & 0 & 1 & 0 & 1 & - \\
\hline P. mirabilis & 0 & 1 & 2 & 3 & 0.630 \\
\hline Total & $40(12)$ & $86(27 \%)$ & 197(61\%) & 323 & \\
\hline
\end{tabular}

Note: $p<0.05$ by Fischer's exact test denotes that significant occurrence of bacterial isolates in a particular group in comparison to two other groups.

common Gram-negative isolates, had 89 and 81\% P-XDR, respectively ( - Table 2 ). Among Gram-positive pathogens, $63 \%$ of isolates were MDR followed by P-XDR (22\%). Only $15 \%$ showed a NMDR pattern. The pattern of drug resistance among different pathogens is summarized in - Table 2 .

It was an alarming observation that approximately 90\% of the isolates were significantly P-XDR among VAP patients ( $p$-value0.01), whereas in CA-UTI isolates MDR patterns were most common ( $p$-value $0.01 \%$ ). Site-specific patterns of antimicrobial resistance are elucidated in - Table 3 .

A. baumannii revealed an alarming resistance pattern with susceptibility to only higher generation antimicrobials like cefoperazone/sulbactam (34.7\%) and colistin (no resistance). K. pneumoniae had also limited susceptibility to high-end antimicrobials only like imipenem (43\%) and cefoperazone/sulbactam (72.9\%) (-Table 4). All Gram-positive isolates were sensitive to linezolid. Methicillin resistance was observed in $70 \%$ of S. aureus isolates. Out of 10 S. aureus isolates, 7 showed methicillin resistance ( 5 from skin and soft tissue infections and 2 from secondary blood stream infection). Notably, about one-third of the Enterococcus faecium isolates were resistant to both teicoplanin and vancomycin (-Table 5). Outcomes were measured in terms of duration of hospital stay and mortality. The median duration of hospital stay among P-XDR was 9 days (2-17 days) and MDR was 17 days (9-13 days), whereas patients with NMDR stayed for 10 days (6-15.5 days). The median duration of P-XDR is less when compared with NMDR and MDR. It is because, P-XDR patients were sick and died earlier. The mortality rate of NMDR, MDR, and P-XDR were 37.5, 42.4, and 72.1\%, respectively.

\section{Antibiogram}

Based on the culture sensitivity pattern, the most effective antibiotic found for VAP was cefoperazone/sulbactam (49\% resistance) followed by imipenem (70.5\% resistance). While symptomatic UTI pathogens showed considerable sensitivity to amikacin and piperacillin/tazobactam, CA-UTI pathogens were highly resistant and sensitive to very few antibiotics like imipenem (84.8\%). Central line-associated BSI (CLABSI) isolates were highly resistant, among which the most effective antibiotic was imipenem (42\%). Antibiotic resistance patterns of culture-positive Gram-negative isolates among the various spectrums of infections are shown in - Table 6 .

\section{Discussion}

The global menace of antimicrobial resistance is further complicated in developing countries like India, owing to a multitude of factors such as the high burden of disease, poor public health infrastructure, lack of appropriate diagnostic support, and paucity of updated and systematic data on the precise estimates of the extent of drug resistance. ${ }^{9}$ This study assessed the prevalence and spectrum of infections caused by MDROs in medicine wards and ICUs of a tertiary care hospital in North India. We came across some noteworthy findings.

First, the microbial agents isolated from the clinical samples from patients of various infectious syndromes recruited in this study are distinct from previously reported studies. We observed a predominance of Gram-negative pathogens (92\% of 323 culture-positive isolates) in our study, which is different from what is reported from western studies. ${ }^{10,11}$ The spectrum of microorganisms responsible for infections in medical ICUs and wards has been reported to be different in western world in comparison to Indian subcontinent. ${ }^{12,13}$ The reason for the same is not fully known, albeit, some believe that this may be due to difference in the climatic condition. ${ }^{12}$ In our study, A. baumannii was the most common bacteria isolated which is in contrast to a 
Table 3 Distribution of drug resistance in different spectrum of infection

\begin{tabular}{|c|c|c|c|c|c|c|}
\hline \multirow[t]{2}{*}{ Sr. no. } & \multirow[t]{2}{*}{ Clinical condition } & \multicolumn{5}{|c|}{ Number (\%) of clinically significant culture isolates } \\
\hline & & NMDR & MDR & P-XDR & Total & \\
\hline \multirow[t]{5}{*}{ A. } & $\begin{array}{l}\text { 1. Respiratory tract } \\
\text { infection }\end{array}$ & 12 & 17 & 110 & $139(43 \%)$ & $p$-value $<0.001$ \\
\hline & 1.1 CAP & 3 & 1 & 0 & 4 & \multirow[t]{4}{*}{-} \\
\hline & 1.2 HAP & 5 & 3 & 24 & 32 & \\
\hline & 1.3 VAP & 0 & 13 & 82 & 95 & \\
\hline & 1.4 Pleural fluid & 4 & 0 & 4 & 8 & \\
\hline \multirow[t]{3}{*}{ B. } & 2. Urinary tract infection & 11 & 41 & 30 & $82(25 \%)$ & $p$-value $=0.01$ \\
\hline & $2.1 \mathrm{~S}-\mathrm{UTI}$ & 8 & 20 & 7 & 35 & \multirow[t]{2}{*}{-} \\
\hline & 2.2 CA-UTI & 3 & 21 & 23 & 47 & \\
\hline \multirow[t]{4}{*}{ C. } & 3. Blood stream infection & 2 & 29 & 42 & $73(22.6 \%)$ & $p$-value $=0.248$ \\
\hline & 3.1 P-BSI & 2 & 13 & 15 & 30 & \multirow[t]{3}{*}{-} \\
\hline & $3.2 \mathrm{~S}-\mathrm{BSI}$ & 0 & 13 & 16 & 29 & \\
\hline & 3.3 CLABSI & 0 & 3 & 11 & 14 & \\
\hline \multirow[t]{5}{*}{ D. } & 4. Others & 5 & 9 & 15 & $29(8 \%)$ & $p$-value $=0.803$ \\
\hline & 4.1 SSTI & 5 & 6 & 10 & 21 & \multirow[t]{4}{*}{-} \\
\hline & 4.2 Meningitis & 0 & 1 & 3 & 4 & \\
\hline & 4.3 Cholangitis & 0 & 1 & 0 & 1 & \\
\hline & 4.4 Peritonitis & 0 & 1 & 2 & 3 & \\
\hline
\end{tabular}

Abbreviations: CAP, community-acquired pneumonia; CA-UTI, catheter-associated urinary tract infection; CLABSI, central line-associated blood stream infection; HAP, hospital-acquired pneumonia; MDR, multidrug resistant; NMDR, nonmultidrug resistant; P-XDR, possible extensive drug resistant; PBSI, primary blood stream infection; S-BSI, secondary blood stream infection; S-UTI, symptomatic urinary tract infection; SSTI, skin and soft tissue infection; VAP, Ventilator associated pneumonia.

Note: $p$-value $<0.001$ by Fisher's exact $t$-test suggesting significant association between occurrence of RTI, UTI, BSI, and others site infection with NMDR, MDR, and P-XDR isolates.

Table 4 Antimicrobial resistance patterns of commonly observed Gram-negative isolates (\%)

\begin{tabular}{|l|l|l|l|l|l|l|l|l|l|l|l|l|}
\hline Sr.no. & Pathogen & Ciplo & Ami & Netli & Cefo & Ceftaz & Cef- Sul & Imi & Mero & Amoxcalv & Piptaz & Colistin \\
\hline 1 & A. baumannii & 98.9 & 98.9 & 89.4 & 100 & 100 & 34.7 & 90.1 & 77.17 & 100 & 98.8 & 0 \\
\hline 2 & K. pneumoniae & 96.5 & 84.8 & 83 & 97.6 & 96.4 & 72.9 & 43 & 83.7 & 100 & 85.71 & 0 \\
\hline 3 & E. coli & 93.6 & 40.5 & 36 & 90.14 & 88 & 39.43 & 24.32 & 80.5 & 90.62 & 86.48 & 0 \\
\hline 5 & P. aeruginosa & 66.6 & 68.2 & 69.2 & 85.71 & 77.27 & 63.63 & 50 & 63.63 & NT & 63.63 & 0 \\
\hline
\end{tabular}

Abbreviations: Ami, amikacin; Amoxcalv, amoxicillin clavulanic acid; Cefo, cefotaxime; Cef-Sul, cefoperazone/sulbactam; Cefta, ceftazidime; Ciplo, ciprofloxacin; Imi, imipenem; Mero, meropenem; Netli, Netlimicin; NT, not tested; Piptaz, piperacillin/tazobactam.

Table 5 Antimicrobial resistance patterns of commonly observed Gram-positive isolates (\%)

\begin{tabular}{|l|l|l|l|l|l|l|l|l|l|l|l|l|}
\hline $\begin{array}{l}\text { Sr. } \\
\text { no. }\end{array}$ & Pathogen & Peni & Amocalv & Ciplox & Eryth & Genta & Ami & Line & Teico & Van & Tetra & Co-Tri \\
\hline 1. & E. faecium & 76.9 & NT & 100 & 100 & 100 & NT & 6.67 & 33.3 & 34.1 & 33.3 & 0 \\
\hline 2. & S. aureus & 77.7 & 66.67 & 70 & 66.67 & 50 & 22.2 & 0 & 0 & 0 & NT & 60 \\
\hline
\end{tabular}

Abbreviations: Ami, amikacin; Amocalv, amoxicillin clavulanic acid; Ciplox, ciprofloxacin; Co-Tri,co-trimoxazole; Eryth, erythromycin; Genta, gentamicin; Line, linezolid; NT, not tested; Peni, penicillin; Teico, teicoplanin; Tetra, tetracycline; Van, vancomycin.

couple of other studies from India where E. coli and K. pneumoniae were reported to be the predominant bacterial isolates from similar settings. ${ }^{13-15} \mathrm{~A}$. baumannii was the most frequently isolated causative pathogen among the patients of VAP. No Gram-positive organism was isolated from respiratory samples. Although, this observation is in agreement with various other Indian studies, but it varies from the reports from western studies that reports a larger number of cases due to Gram-positive bacteria. ${ }^{16}$ In our study, the most common isolate causing BSI was K. pneumoniae (30\%), followed by A. baumannii (23.2\%). E. faecium was the most common Gram-positive isolate (8.2\%). These findings are different from a couple of other studies published earlier from the Indian setting which has reported Staphylococcus spp., A. baumannii, and E. coli as the most common organisms. ${ }^{17,18}$ The microbial profiles in various diseases keep on 
Table 6 Antibiotic resistance patterns of common Gram-negative isolates among various spectrums of infections (\%)

\begin{tabular}{|l|l|l|l|l|l|l|l|l|l|l|l|l|}
\hline Sr. no. & Infection & Ciplo & Ami & Netli & Cefo & Ceftaz & Cef-Sul & Imi & Mero & Amoxclav & Piptaz & Colistin \\
\hline 1. & CAP & 75 & 25 & 25 & 100 & 50 & 0 & 0 & 25 & NT & 0 & NT \\
\hline 2. & HAP & 87 & 81 & 62 & 100 & 93.75 & 40 & 69 & 81 & 100 & 78 & 0 \\
\hline 3. & VAP & 98 & 96 & 92 & 100 & 98 & 49 & 70.5 & 78 & 89 & 96 & 0 \\
\hline 4. & S-UTI & 89.2 & 23.3 & 31 & 85.2 & 86.6 & 27.5 & 20.6 & 68.6 & 82.3 & 58.6 & NT \\
\hline 5. & CA-UTI & 97.7 & 62.6 & 62.8 & 94.4 & 91.8 & 59.4 & 16.2 & 86.4 & 100 & 86.4 & 0 \\
\hline 6. & P-BSI & 96 & 73 & 60 & 100 & 100 & 67 & 50 & 86 & 100 & 87 & 0 \\
\hline 7. & S-BSI & 85 & 100 & 67 & 67 & 90 & 59 & 88 & 882.3 & 100 & 94 & 0 \\
\hline 8. & CLABSI & 100 & 91 & 100 & 100 & 100 & 92 & 58 & 100 & 100 & 92 & 0 \\
\hline
\end{tabular}

Abbreviations: Ami, amikacin; Amoxcalv, amoxicillin clavulanic acid; CAP, community-acquired pneumonia; CA-UTI, catheter-associated urinary tract infection; CLABSI, central line-associated blood stream infection; Cefo, cefotaxime; Cef-Sul, cefoperazone/sulbactam; Ceftaz- ceftazidime; Ciplo, ciprofloxacin; HAP, hospital-acquired pneumonia; Imi, imipenem; Mero, meropenem; Netli, Netlimicin; NT, not tested; P-BSI, primary blood stream infection; Piptaz, piperacillin/ tazobactam; S-BSI, secondary blood stream infection; S-UTI, symptomatic urinary tract infection; VAP, ventilator-associated pneumonia.

varying from time to time and region to region depending on various host and institutional factors.

The burden of MDROs among patients admitted with infectious diseases has reached an alarming level in this part of the world. About $90 \%$ of the culture isolates had significant drug resistance with 60\% having P-XDR. Approximately 90\% of the cases of VAP and CLABSI had infection due to a P-XDR organism. A. baumannii and $K$. pneumoniae, the two most common bacteria isolated, had shown the prevalence of P-XDR in almost 90 and $80 \%$ cases, respectively. The high prevalence of drug resistance emphasizes the importance of appropriate antibiotic usage and implementation of antibiotic stewardship programs. It also mandates the need for continuous surveillance and generation of updated data for the effective use of antibiotics to prevent further spread of resistance.

The high prevalence of MDROs has left physicians with few options for empirical choice of antibiotics for most of the critical illnesses in this part of the world. Cefoperazone/ sulbactam and imipenem seems to be the only antibiotics useful as empirical choice antibiotics for VAP. It is also in agreement with several recent studies. ${ }^{19,20}$ Much limited option is left for secondary BSI (cefoperazone/sulbactam and colistin) and CLABSI (imipenem and colistin). These findings were similar to other studies conducted in our region. ${ }^{21}$ With the rise in isolation of MDR Gram-negative organisms, the need for alternative treatments has led to the resurgence of colistin use as also observed in our study. Although colistin has been shown to be effective for the treatment of a wide variety of infections, the development of colistin resistance is a serious concern..$^{22}$ Fortunately, none of the isolates from our study showed colistin resistance.

This study is one of the few large-scale prospective studies, especially from the Indian subcontinent, that assessed the prevalence and spectrum of infections caused by MDROs. ${ }^{14,23}$ The strength of this study is the inclusion of a large number of culture-positive isolates and focus on antimicrobial resistance patterns in the context of site-specific infections. There are a few limitations of this study. One of them is the inclusion and formulation of an antibiogram on culture-positive drug-resistant isolates alone, which may not be a true representation of drug-resistant organisms due to limited culture positivity. Also, numbers of isolates from community-acquired infections were limited probably owing to tertiary level hospital settings and referral bias, and hence findings/observations from small number of community isolates cannot be extrapolated to other settings reiterating the need of further large-scale studies specially focusing on antibiogram in community infections.

\section{Conclusion}

This study provides an insight into the local resistance patterns and prevalence of drug-resistant organisms in a different spectrum of infections to further guide appropriate antibiotic prescription practices and to channelize robust antibiotic stewardship programs. This study clearly shows the epidemiology of antibiogram varies from western countries to developing countries like India. Updated and regular surveillance on the burden of drug resistance is essential to guide clinicians in the choice of appropriate empiric antimicrobial leading to a decrease in overall resistance rates and also achieve better clinical outcomes. Sound knowledge of local epidemiology and antibiogram of bacterial isolates will help in designing an adequate and appropriate empirical cover and antibiotic stewardship practices.

\section{Conflict of Interest}

None declared.

\section{References}

1 World Health Organization. New report calls for urgent action to avert antimicrobial resistance crisis. Available at: https:// www.who.int/news/item/29-04-2019-new-report-callsfor-urgent-action-to-avert-antimicrobial-resistance-crisis. Accessed October 23, 2020.

2 Kadri SS, Adjemian J, Lai YL, et al; National Institutes of Health Antimicrobial Resistance Outcomes Research Initiative (NIH-ARORI). Difficult-to-treat resistance in Gram-negative bacteremia at 173 US hospitals: retrospective cohort analysis of prevalence, predictors, and outcome of resistance to all first-line agents. Clin Infect Dis 2018;67(12):1803-1814

3 Aslam B, Wang W, Arshad MI, et al. Antibiotic resistance: a rundown of a global crisis. Infect Drug Resist 2018;11:1645-1658 
4 Laxminarayan R, Chaudhury RR. Antibiotic resistance in India: drivers and opportunities for action. PLoS Med 2016;13(3):e1001974

5 Halstead DC, Gomez N, McCarter YS. Reality of developing a community-wide antibiogram.JClin Microbiol 2004;42(1):1-6

6 Weinstein MP. Performance Standards for Antimicrobial Susceptibility Testing.Wayne, PA:Clinical and Laboratory Standards Institute;2019

7 Horan TC, Andrus M, Dudeck MA. CDC/NHSN surveillance definition of health care-associated infection and criteria for specific types of infections in the acute care setting. Am J Infect Control 2008;36(5):309-332

8 Magiorakos A-P, Srinivasan A, Carey RB, et al. Multidrugresistant, extensively drug-resistant and pandrug-resistant bacteria: an international expert proposal for interim standard definitions for acquired resistance. Clin Microbiol Infect 2012;18(3):268-281

9 Dixit A, Kumar N, Kumar S, Trigun V. Antimicrobial resistance: progress in the decade since emergence of New Delhi metallo- $\beta$-lactamase in India. Indian J Community Med 2019; 44(1):4-8

10 Chaudhry D, Prajapat B. Intensive care unit bugs in India: how do they differ from the Western world? J Assoc Chest Physicians 2017;5(1):10

11 Venkataraman R, Divatia JV, Ramakrishnan N, et al. Multicenter observational study to evaluate epidemiology and resistance patterns of common intensive care unit-infections. Indian J Crit Care Med 2018;22(1):20-26

12 Schwab F, Gastmeier P, Meyer E. The warmer the weather, the more gram-negative bacteria - impact of temperature on clinical isolates in intensive care units. PLoS One 2014;9(3):e91105

13 Veeraraghavan B, Jesudason MR, Prakasah JA, et al. Antimicrobial susceptibility profiles of gram-negative bacteria causing infections collected across India during 2014-2016: study for monitoring antimicrobial resistance trend report. Indian J Med Microbiol 2018;36(1):32-36
14 Basak S, Singh P, Rajurkar M. Multidrug resistant and extensively drug resistant bacteria: a study. J Pathogens 2016; 2016:4065603

15 Gandra S, Tseng KK, Arora A, et al. The mortality burden of multidrug-resistant pathogens in India: a retrospective, observational study. Clin Infect Dis 2019;69(4):563-570

16 Cilloniz C, Martin-Loeches I, Garcia-Vidal C, San Jose A, Torres A. Microbial etiology of pneumonia: epidemiology, diagnosis and resistance patterns. Int J Mol Sci 2016;17(12):2120

17 Singh A, Venkatesh V, Singh R, Singh M. Bacterial and antimicrobial resistance profile of bloodstream infections: ahospital-based study. CHRISMED J Health Res 2014;1(3):140

18 Vasudeva N, Nirwan PS, Shrivastava P. Bloodstream infections and antimicrobial sensitivity patterns in a tertiary care hospital of India. Ther Adv Infect Dis 2016;3(5):119-127

19 Gupta N, Soneja M, Ray Y, et al. Nosocomial pneumonia: search for an empiric and effective antibiotic regimen in high burden tertiary care centre. Drug Discov Ther 2018;12(2):97-100

20 Patro S, Sarangi G, Das P, et al. Bacteriological profile of ventilator-associated pneumonia in a tertiary care hospital. Indian J Pathol Microbiol 2018;61(3):375-379

21 Gupta S, Kashyap B. Bacteriological profile and antibiogram of blood culture isolates from a tertiary care hospital of North India. Trop J Med Res 2016;19(2):94

22 Elbediwi M, Li Y, Paudyal N, et al. Global burden of colistin-resistant bacteria: mobilized colistin resistance genes study (1980-2018) Microorganisms 2019;7(10):461

23 Siwakoti S, Subedi A, Sharma A, Baral R, Bhattarai NR, Khanal B. Incidence and outcomes of multidrug-resistant gram-negative bacteria infections in intensive care unit from Nepal- a prospective cohort study. Antimicrob Resist Infect Control 2018;7(1):114 